\title{
Older adults with acute myeloid leukemia treated with intensive chemotherapy: "old" prognostic algorithms may not apply
}

\author{
Richard M. Stone and Coleman Lindsley
}

Department of Medical Oncology, Dana-Farber Cancer Institute, Boston, MA, USA

\section{E-mail: rstone@partners.org}

doi:10.3324/haematol.2018.201848

T The notion that older adults with acute myeloid leukemia (AML) represent a major therapeutic challenge due to host biological factors and intrinsic disease features leading to poor outcomes is accepted by consensus. Older adults have diminished stem cell reserve, more frequent comorbid medical conditions, and decreased ability to excrete chemotherapy compared to younger patients, in part leading to a relatively increased rate of mortality from induction treatment. ${ }^{1}$ Moreover, AML that arises in the older population can exhibit features of intrinsic chemo-resistance exemplified by a higher expression of proteins that extrude chemotherapy, a higher proportion of adverse cytogenetic abnormalities, ${ }^{2}$ and a higher frequency of gene mutations associated with antecedent myeloid disease, which are, in turn, associated with an elevated rate of disease resistance. ${ }^{3}$ Based on this unfavorable balance of response and toxicity, many believe that older patients, especially those over the age of 75 , should as a whole be treated with less intensive therapies: usually a hypomethylating agent (azacytidine or decitabine) in the USA or lowdose cytarabine. ${ }^{4}$ In counterpoint, a population-based study from Sweden suggested that older adults may benefit from intensive treatment, while others have shown that the genetic characteristics ${ }^{5}$ of AML in older patients may discriminate subgroups with distinct clinical responses to intensive therapy. ${ }^{3}$ For example, patients with de novo AML genetic alterations have a higher likelihood of responding to induction therapy, while both 'secondary-type' AML mutations (those associated with AML arising after a prodromic myelodysplastic syndrome) and TP53 mutations are associated with more resistant disease. ${ }^{3}$ The challenge facing the field is how to incorporate contrasting data from large studies of heterogeneous patient populations that may lack sufficient data with regards to genetic variables and smaller studies with detailed clinical information and genetic annotation, but limited statistical power to identify independent genetic effects. The debate about ' $3+7$ ' chemotherapy versus non-intensive therapy for older adults with AML continues. ${ }^{6}$

How do the data provided by the German Austrian AML Study Group ${ }^{7}$ add to the discussion? Their study has several important strengths. First, only patients older than the age of 74 were included, enabling a distinct and focused evaluation of this under-studied population. Second, all patients received the same intensive induction chemotherapy on an AML Cooperative Group protocol, ${ }^{8}$ which compared standard induction chemotherapy with thioguanine, daunorubicin, and cytarabine (TAD9) with a high-dose cytarabine/mitoxantrone induction. Patients whose bone marrow samples showed residual AML on day 15 were to receive high-dose cytarabine/mitoxantrone. The doses of chemotherapy were attenuated in older adults. Third, the authors performed targeted, next-generation sequencing of 64 genes known to be mutated in AML, paired with focused assessment of FLT3-ITD, NPM1, and CEBPA mutations by standard laboratory-validated methodologies. They looked at outcomes according to number and type of mutations along with other potentially relevant data such as patient's age, cytogenetics, risk group by one of two classifications systems, and randomized treatment assignment. More details about why these older adults received chemotherapy as opposed to other therapies would have been welcome. Were they particularly fit? Some actually had a performance status worse than 2 , which is an adverse prognostic factor for response to chemotherapy. ${ }^{2}$ Obviously, no comparative group of patients treated contemporaneously with less intensive chemotherapy can be provided.

The analysis highlights that some commonly employed prognostic factors in younger adult AML patients, including FLT3-ITD and NPM1, did not have the same relevance in the older patients. The European LeukemiaNet ${ }^{9}$ and Medical Research Counci ${ }^{10}$ prognostic algorithms simply were not developed based on data from intensively treated patients in this age group. Given the distinct clinical and molecular features in this age group, one can begin to grasp why the 'usual prognostic rules' noted in younger patients may not apply. The findings in this cohort of 151 patients aged over 74 years old confirm those of published studies showing that there is a preponderance of gene mutations associated with myelodysplastic syndromes and clonal hematopoiesis of indeterminate potential in AML in older individuals. ${ }^{3,11,12}$ In a previous study, outcomes after 3+7based chemotherapy were particularly poor in patients with histologically confirmed secondary AML or de novo AML harboring myelodysplastic syndrome-associated gene mutations. ${ }^{3}$ Although few patients in this study were older tha 74 , it raised the idea that gene mutations suggestive of antecedent myelodysplastic syndromes (SRSF2, SF3B1, U2AF1, ZRSR2, BCOR, EZH2, ASXL1, STAG2) may highlight a subgroup of older patients with more chemoresistant disease. By contrast, older patients without such mutations had a much more favorable induction success rate and a $50 \%$ event-free survival. ${ }^{3}$

In the cohort reported by Prassek et al., $35 \%$ of the patients died during induction. This finding has important clinical implications, as older patients among a group of seemingly fit-for-chemotherapy patients may experience elevated treatment-related mortality due to a poor stem cell reserve, subtle organ dysfunction, and increased co-morbid disease, which may sometimes overwhelm the effect of disease biology. This finding offers important context for the interpretation of genetic results. In $\mathrm{AML}$, the most prominent and reproducible associations between specific 
gene mutations (FLT3, TP53, CEBPA, NPM1) and overall survival are related to the mutations' differential impact on relapse risk. ${ }^{13}$ In fact, there are limited data available regarding the potential association between disease genetics and induction mortality. Notably, ten out of 13 patients with IDH1 mutations experienced early death during induction, while just two had refractory disease. This detail is central to the interpretation of the results and to contemplating clinical actionability. Do IDH1 mutations drive specific chemoresistance in older patients, thereby suggesting augmentation of induction with IDH1-directed therapy? Are IDH1 mutations causally or non-causally associated with clinical features that have been linked to increased transplant-related mortality? ${ }^{14}$ A combination of the two? In younger adults the prognostic impact of IDH mutations is unclear. One study suggested that those with both NPM1 and IDH mutations enjoyed a high event-free survival rate when treated with aggressive chemotherapy ${ }^{15}$ while others showed a neutral or negative impact. ${ }^{16,17}$ The simplest explanation for the disparate literature regarding IDH1 mutations is that related to the random noise in small samples. Alternatively, the clinical impact of IDH mutations may be 'context-dependent', and different depending on its place in the clonal hierarchy (progression mutation in some cases and a founder or 'early' mutation in others), the co-occurring gene mutations, or undefined clinical characteristics. Does this group of patients in their 70's and 80's have IDH1 mutations that reflect a distinct group with particularly chemoresistant disease? If so, one could speculate that such individuals might have fared better with an IDH inhibitor than with chemotherapy. Indeed the single-agent studies with these drugs, ${ }^{18,19}$ mainly conducted in patients with advanced disease, include some previously untreated patients with comparable outcomes.

In summary, the German Austrian AML Study Group research is important because it confirms the commonality of secondary or stem cell type mutations in adults aged over 74 with AML. It further reminds us that prognostic characteristics cannot necessarily be applied with abandon across the age spectrum. However, we remain in the same conundrum about the utility of aggressive versus less intensive therapy in this age group.

\section{References}

1. Klepin HD, Geiger AM, Tooze JA, et al. Geriatric assessment predicts survival for older adults receiving induction chemotherapy for acute myelogenous leukemia. Blood. 2013;121(21):4287-4294.

2. Leith CP, Kopecky KJ, Godwin J, et al. Acute myeloid leukemia in the elderly: assessment of multidrug resistance (MDR1) and cytogenetics distinguishes biologic subgroups with remarkably distinct responses to standard chemotherapy. A Southwest Oncology Group study. Blood. 1997;89(9):3323-3329.

3. Lindsley RC, Mar BG, Mazzola E, et al. Acute myeloid leukemia ontogeny is defined by distinct somatic mutations. Blood. 2015;125(9):1367-1376.

4. Ferrara F, Barosi G, Venditti A, et al. Consensus-based definition of unfitness to intensive and non-intensive chemotherapy in acute myeloid leukemia: a project of SIE, SIES and GITMO group on a new tool for therapy decision making. Leukemia. 2013;27(5):997-999.

5. Juliusson G, Antunovic P, Derolf A, et al. Age and acute myeloid leukemia: real world data on decision to treat and outcomes from the Swedish Acute Leukemia Registry. Blood. 2009;113(18):4179-4187.

6. Ossenkoppele G, Lowenberg B. How I treat the older patient with acute myeloid leukemia. Blood. 2015;125(5):767-774.

7. Prassek VV, Rothenberg-Thurley M, Sauerland MC, et al. Genetics of acute myeloid leukemia in the elderly: mutation spectrum and clinical impact in intensively treated patients aged 75 years or older. Haematologica. 2018;103(11):1853-1861.

8. Buchner T, Berdel WE, Haferlach C, et al. Long-term results in patients with acute myeloid leukemia (AML): the influence of high-dose AraC, G-CSF priming, autologous transplantation, prolonged maintenance, age, history, cytogenetics, and mutation status. Data of the AMLCC 1999 trial. Blood. 2009;114(22):485

9. Döhner H, Estey E, Grimwade D, et al. Diagnosis and management of AML in adults: 2017 ELN recommendations from an international expert panel. Blood. 2017;129(4):424-447.

10. Grimwade D, Hills RK, Moorman AV, et al. Refinement of cytogenetic classification in acute myeloid leukemia: determination of prognostic significance of rare recurring chromosomal abnormalities among 5876 younger adult patients treated in the United Kingdom Medical Research Council trials. Blood. 2010;116(3):354-365.

11. Papaemmanuil E, Gerstung M, Bullinger L, et al. Genomic classification and prognosis in acute myeloid leukemia. $N$ Engl J Med. 2016;374(23):2209-2221.

12. Metzeler KH, Herold T, Rothenberg-Thurley M, et al. Spectrum and prognostic relevance of driver gene mutations in acute myeloid leukemia. Blood. 2016;128(5):686-698.

13. Marcucci G, Haferlach T, Döhner H. Molecular genetics of adult acute myeloid leukemia: prognostic and therapeutic implications. J Clin Oncol. 2011:29(5):475-486.

14. Walter RB, Othus M, Borthakur G, et al. Prediction of early death after induction therapy for newly diagnosed acute myeloid leukemia with pretreatment risk scores: a novel paradigm for treatment assignment. J Clin Oncol. 2011;29(33):4417-4423.

15. Patel JP, Gönen M, Figueroa ME, et al. Prognostic relevance of integrated genetic profiling in acute myeloid leukemia. N Engl J Med. 2012;366(12):1079-1089.

16. Green CL, Evans CM, Zhao L, et al. The prognostic significance of IDH2 mutations in AML depends on the location of the mutation. Blood. 2011;118(2):409-412

17. Paschka P, Schlenk RF, Gaidzik VI, et al. IDH1 and IDH2 mutations are frequent genetic alterations in acute myeloid leukemia and confer adverse prognosis in cytogenetically normal acute myeloid leukemia with NPM1 mutation without FLT3 internal tandem duplication. J Clin Oncol. 2010;28(22):3636-3643.

18. Stein EM, DiNardo CD, Pollyea DA, et al. Enasidenib in mutant IDH2 relapsed or refractory acute myeloid leukemia. Blood. 2017;130(6):722731.

19. DiNardo CD, Stein EM, de Botton S, et al. Durable remissions with ivosidenib in IDH1-mutated relapsed or refractory AML. N Engl J Med. 2018;378(25):2386-2398. 